\title{
Die kreative Projektarbeit - Fachkräftesicherung in Brandenburg
}

JÖRG JURKEIT

\section{Fachkräfte sichern}

Die deutsche Volkswirtschaft muss sich in den nächsten Jahrzehnten zunehmend auf die Bedingungen eines zurückgehenden und alternden Arbeitskräfteangebotes einstellen. Gegenwärtig befinden sich deutschlandweit knapp 50 Mio. Menschen im erwerbsfähigen Alter zwischen 20 und 64 Jahren. Ab 2015 wird sich diese Zahl deutlich verringern. Berechnungen des Statistischen Bundesamtes zeigen, dass sich bis 2030 das Arbeitskräftepotenzial um rund 6,3 Mio. Menschen reduzieren wird. ${ }^{\bullet}$ Ferner wird sich mit der Alterung der besonders geburtenstarken Jahrgänge 1955 bis 1964 der Altersdurchschnitt in den Belegschaften deutlich erhöhen. In Anbetracht dieser Entwicklungen gewann im letzten Jahr die Diskussion um die Sicherung des Fachkräftebedarfs neuen Schwung. Beispielsweise beschloss die Bundesregierung im Juni 2011 ein Konzept zur Fachkräftesicherung, das die Erhöhung der Erwerbsbeteiligung und des Qualifikationsniveaus der Bevölkerung anstrebt. Insgesamt werden fünf Sicherungspfade beschrieben. Neben der Stärkung der Bildungschancen für alle sowie der Förderung der Qualifizierung und Aus- und Weiterbildung gehören hierzu: die bessere Vereinbarkeit von Familie und Beruf, die Beschäftigungssicherung (insbesondere von älteren Arbeitnehmern), eine qualifizierte Zuwanderung von Arbeitskräften und die Aktivierung und Integration von Arbeitslosen. ${ }^{2}$ Dabei wird der Nutzung und Förderung inländischer Potenziale in der Fachkräftesicherungspolitik ein Vorrang eingeräumt. Betont wird aber auch, dass mit Blick auf den demografischen Wandel zudem verstärkt auf eine qualifizierte Zuwanderung gesetzt werden müsse. ${ }^{3}$

\section{Ältere arbeitslose Akademiker integrieren}

Die Arbeitspolitik sollte alle Pfade verfolgen. Es lohnt sich jedoch, einen speziellen Blick auf bereits vorhandene und bislang für den Arbeitsmarkt ungenutzte Potenziale zu richten. So sind deutschlandweit rund 60.000 über 50-jährige Akademiker arbeitslos. ${ }^{\circledR}$ Sie suchen eine Stelle und wollen ihre Fähigkeiten und Kenntnisse in die Arbeitswelt einbringen, haben jedoch in formalen Bewerbungsverfahren gegenüber jüngeren Mitbewerbern in der Regel das Nachsehen. Selbst bei hohem Qualifikationsniveau ist es für Ältere sehr schwer, aus der Arbeitslosigkeit wieder in Be- schäftigung zu kommen. ${ }^{\ominus}$ Dabei stellen ältere arbeitslose Akademiker eine wichtige Fachkräftereserve für den deutschen Arbeitsmarkt dar, die bislang zu wenig genutzt wird.

Im Folgenden möchte ich einen im Land Brandenburg erprobten und Erfolg versprechenden Weg zur Aktivierung und Integration von älteren arbeitslosen Akademikern vorstellen, der das Potenzial für eine bundesweite Umsetzung hat. Die Ergebnisse und Aussagen beruhen hauptsächlich auf Untersuchungen, die das Institut für Sozialforschung und Sozialwirtschaft e. V. (iso-Institut) im Rahmen einer wissenschaftlichen Begleitung durchgeführt hat.

\section{Ein Weg zur Integration: die kreative Projektarbeit}

Im Rahmen der Brandenburger Modellförderung INNOPUNKT startete im Dezember die Initiative „Ältere - Erfahrung trifft Herausforderung “ mit dem Ziel, innovative Lösungen zu finden, die die Beschäftigungschancen älterer Menschen verbessern. Drei Modellprojekte boten dabei unter der Überschrift „Kreative Projektarbeit“ neue praxisbezogene Lernarrangements für ältere arbeitslose Akademiker und Fachkräfte in Kooperation mit Hochschulen und Unternehmen an. Die Initiative endete nach dreijähriger Laufzeit im Dezember 2011.

An der Erprobung des Ansatzes „Kreative Projektarbeit“ (KPA) waren mit der Brandenburgischen Technischen

\footnotetext{
Bundesregierung (2011): Bericht der Bundesregierung zur demografischen Lage und künftigen Entwicklung des Landes, Oktober, Berlin, S. 105.

(2) Bundesregierung (2011): Fachkräftesicherung - Ziele und Maßnahmen der Bundesregierung, Juni, Berlin.

3 Vgl. ebd., S. 32.

(4) Statistik der Bundesagentur für Arbeit, Jahresdurchschnitt 2010.

(5) Die Eintrittschancen Älterer aus der Arbeitslosigkeit in den Arbeitsmarkt sind erheblich schlechter als die der Jüngeren. Das zeigen z. B. Untersuchungen zu Eintrittsraten Älterer in Beschäftigung. Siehe hierzu Brussig, M. (2011): Neueinstellungen im Alter:Tragen sie zu verlängerten Erwerbsbiografien bei?, Altersübergangs-Report 2011-03.
} 
Universität (BTU) Cottbus, der Hochschule für nachhaltige Entwicklung (HNE) Eberswalde, der Europa-Universität Viadrina Frankfurt (Oder) und der Universität Potsdam vier Brandenburger Hochschulen beteiligt. Insgesamt nahmen 207 arbeitslose Akademiker und Fachkräfte über 50 Jahre (davon 113 Männer und 94 Frauen) an der KPA teil. Bemerkenswert ist, dass zwei Drittel der Teilnehmenden bereits über 55 Jahre alt waren. An der Universität Potsdam arbeiteten die Älteren gemeinsam mit Studierenden an den Projektaufgaben.

In Teams wurden 53 Projektarbeiten zusammen mit 50 Betrieben bearbeitet. 87 Teilnehmenden (42\%) gelang der Sprung zurück in die Erwerbstätigkeit (inkl. Mini-Jobs und Selbstständigkeit); davon konnten 65 Personen eine sozialversicherungspflichtige Beschäftigung aufnehmen. Neben dem beachtlichen Vermittlungserfolg erreichte die KPA auch einen deutlichen Motivationsschub bei den Teilnehmenden; selbst bei den Nichtvermittelten. Eine repräsentative schriftliche Teilnehmerbefragung zeigt, dass 47,7 \% derjenigen, die keine neue Anstellung finden konnten, dennoch wieder optimistischer in ihre berufliche Zukunft blicken. 80,3\% aller Teilnehmenden würden wieder an der KPA teilnehmen. Das unterstreicht, dass die KPA als praxisbezogene Qualifizierungsform im Sinne des handelnden bzw. forschenden Lernens die Älteren besonders gut angesprochen hat. Auch bei den beteiligten Unternehmen stieß die KPA auf eine hohe Akzeptanz. Alle befragten Unternehmen befürworten eine Neuauflage des Angebotes. Bislang gelang die Weiterführung der KPA im Landkreis Barnim im Rahmen der Landesförderung „Regionalbudget“.

\section{Die KPA-Methode}

Im Kern werden bei der KPA konkrete betriebliche Aufgaben von älteren erwerbslosen Akademikern und Fachkräften im Team bearbeitet. In einer Partnerschaft arbeiten dabei Hochschulen, Weiterbildungsträger und Unternehmen zusammen. Die Hochschulen geben methodische und fachliche Unterstützung auf neuestem wissenschaftlichen Niveau. Träger organisieren den Lernprozess und sichern die Begleitung der Projektteams. Die Unternehmen formulieren praxisrelevante Probleme und stellen betriebliche Ressourcen zur Verfügung. Im Ergebnis der Projektarbeit entsteht ein Produkt, z. B. ein Konzept, ein Entwurf, eine Computeranimation, eine Dokumentation, eine Marktanalyse. Unterm Strich ist die KPA eine anwendungsbezogene Qualifizierungsform, durch die Ältere ihre Leistungsfähigkeit zeigen können. Die Besonderheit der KPA liegt darin, dass eine neuartige Beziehung zwischen Betrieben, arbeitslosen Akademikern und Hochschulen hergestellt wird, in der innovative betriebliche Aufgabenstellungen mit Unterstützung der Hochschulen bearbeitet werden, sodass die älteren Arbeitslosen ihr vorhandenes Innovations- und Kreativpotenzial unter Beweis stellen können.

Der KPA-Ansatz nützt sowohl den Arbeitslosen als auch den kooperierenden Arbeitgebern:
- Für die arbeitslosen Akademiker ist die KPA eine Gelegenheit, zu zeigen, was sie können und dass sie noch nicht zum alten Eisen gehören.

- Für die Arbeitgeber ist sie eine Chance, den Wert der älteren Arbeitskraft wieder schätzen zu lernen und Projekte in Angriff zu nehmen, die ansonsten in der Schreibtischschublade geblieben wären.

\section{Drei Formen der KPA}

Im Laufe der Förderung haben sich drei Formen bzw. Schwerpunkte in der Umsetzung der KPA herauskristallisiert:

(1) Der betriebsorientierte Ansatz in Frankfurt (Oder) und Cottbus (Träger: Kooperationsstelle Wissenschaft und Arbeitswelt - KOWA) widmete dem erfolgreichen Matching zwischen Teilnehmenden und Betrieben große Aufmerksamkeit und nahm für die Betriebe eine Vorauswahl bei den teilnehmenden Arbeitslosen vor. Die Projektarbeit fand überwiegend in den Betrieben statt. Zudem wurde durch den Abschluss eines Drei-Parteien-Vertrages zwischen Teilnehmer, Betrieb und Weiterbildungsträger eine hohe Verbindlichkeit im Handeln erreicht. Man vereinbarte festgelegte Arbeitszeiten, eine Aufwandsentschädigung und betriebliche Ansprechpersonen und Ressourcen.

(2) Der hochschulbezogene Ansatz in Potsdam (Träger: UPTransfer, Gesellschaft für Wissens- und Technologietransfer) stellte eine enge Bindung zum akademischen Betrieb her. Die Teilnehmenden der KPA arbeiteten gemeinsam in altersgemischten Teams mit Studierenden und besuchten als Gasthörer reguläre Vorlesungen der Universität.

(3) Der teilnehmerbezogene Ansatz in Eberswalde (Träger: Akademie 2. Lebenshälfte) legte großen Wert auf die Einbeziehung der KPA-Teilnehmenden in den gesamten Projektverlauf. Ein besonderes Merkmal dieses Ansatzes war, dass die Teilnehmenden bereits den Prozess der Projektauswahl gestalteten und schließlich auch die konkrete Projektaufgabe ausformulierten.

\section{Betriebe und Kreativprojekte}

Die kooperierenden Betriebe stellten die Projektaufgaben, unterstützten die Projektarbeit fachlich und nahmen die Projektergebnisse entgegen. Da die Kreativprojekte einen unmittelbaren Praxisbezug haben sollten, waren die Betriebe das Kernstück der KPA. Gut die Hälfte der Kooperationsbetriebe waren Kleinstbetriebe mit weniger als zehn Beschäftigten, und ca. ein Drittel waren Kleinunternehmen mit zehn bis 49 Beschäftigten. Dabei engagierten sich am häufigsten Unternehmen aus dem Dienstleistungssektor (65 \%) in der KPA. Zudem waren Hightech-Unternehmen mit einem Anteil von $27 \%$ gut vertreten. Klassische Industriebetriebe sowie das produzierende Gewerbe (insgesamt 8 \%) spielten in den Projekten kaum eine Rolle. 
Im Laufe der Projektumsetzung ließen sich vier Motive zur Teilnahme bei den Betrieben identifizieren:

(1) Kleine Betriebe, die sich noch im Aufbau befinden und alle personellen Ressourcen für das Tagesgeschäft aufwenden müssen, können über die KPA weitergehende Fragen bearbeiten lassen, die sonst vernachlässigt werden müssen.

(2) Bei stark wachsenden Unternehmen mit einem hohen strukturellen Veränderungsdruck vermehren sich die internen Schnittstellen. Die KPA kann bei der Reflexion der Binnenorganisation unterstützen.

(3) Öffentliche Dienstleister stehen vor dem Hintergrund des demografischen Wandels vor zahlreichen neuen Herausforderungen. Sie müssen ihre Aktivitäten zur Daseinsvorsorge neu justieren und haben hierzu einen hohen Informationsbedarf. Die KPA kann über Recherchearbeiten und Hintergrundwissen unterstützend tätig sein.

(4) Hightech-Unternehmen, die sich nicht sicher sind, ob ihre neuen Innovationen ausreichende Realisierungs- und Erfolgsaussichten haben, benötigen Entscheidungsparameter und Marktanalysen, zu denen die KPA beitragen kann.

Unterteilt nach Themenschwerpunkten nahmen die Projektarbeiten, die sich mit Marketing (63\%) beschäftigten, den größten Raum ein. 22 \% der Arbeiten befassten sich mit technischen, $15 \%$ mit organisatorischen Fragen. Unter den Marketingthemen spielten besonders Marktanalysen eine Rolle. Bei den Technikthemen waren Lösungen im Energieund Umweltbereich gefragt, und bei den Organisationsthemen dominierten KPA-Projekte zu Organisationsaufbau und Strukturoptimierung. In allen drei Themenfeldern wurden Recherchearbeiten und Dokumentenanalysen sowie empirische Untersuchungen mit eigenen Befragungen durchgeführt. Die Informationen wurden verdichtet und aufgearbeitet, sodass die Projektergebnisse vor den Unternehmen präsentiert werden konnten.

Beispiele für Projektthemen waren:

- Realisierung der Markteinführung einer modernen Software in öffentlichen Bibliotheken,

- Dienstleistungen für ein langes Leben - Bedarfe und Motive von Senioren für die Inanspruchnahme von professionellen haushaltsnahen Dienstleistungen,

- Studie zur Senkung des Primärenergiebedarfs an Backöfen in Bäckereibetrieben,

- Campingpark Sanssouci - Ermittlung von Maßnahmen zur klimaneutralen Parkbewirtschaftung,

- Erarbeitung von Ausbildungsunterlagen zu einem didaktischen Modell im Fachbereich Mechatronik,

- Erstellung einer allgemeingültigen Anlagendokumentation für Solarmodule.
In der Regel ist es nicht einfach, betriebliche Kooperationspartner für Projekte der Arbeitsmarktpolitik zu gewinnen. Da mit der Förderung echte betriebliche Themen berührt wurden und mit den beteiligten Hochschulen attraktive Partner beteiligt waren, gelang die Unternehmensakquisition in den KPA-Projekten jedoch erstaunlich gut. Der Nutzen für die kooperierenden Unternehmen konnte gut vermittelt werden. Dabei war es aber wichtig, dass die Projektpartner von Anfang an auf einen fairen Austausch zwischen Unternehmen und Teilnehmenden achteten. Als vorteilhaftes Instrument hierzu hat sich die Unterzeichnung eines DreiParteien-Vertrages bewährt. Dieser könnte sinnvoll durch eine verbindliche Beteiligung der Betriebs- und Personalräte ergänzt werden. Zudem liegt es als Ausgestaltungsoption nahe, die älteren Beschäftigten in die Qualifizierung mit einzubeziehen. Somit würden auch die Mitarbeiter der Kooperationsbetriebe von der KPA profitieren können.

\section{Schlussfolgerungen}

Um ältere, hochgebildete Arbeitslose wieder in Arbeit zu bringen, wurden Hochschulen, Betriebe und Weiterbildungsträger miteinander vernetzt. Dieses Akteursdreieck bildet den Grundstein für eine erfolgreiche Unterstützung von älteren arbeitslosen Akademikern. Darüber hinaus lassen sich im Einzelnen vier Erfolgsfaktoren der KPA feststellen.

Erstens ist hier der hohe Praxisbezug zu nennen, der durch die enge Anbindung der Projektarbeit an die Unternehmen gewährleistet werden konnte. Für die Erhöhung der Integrationschancen sowie für die Lernmotivation der Teilnehmenden war es wichtig, dass die Projektarbeit mit konkreten betrieblichen Problemstellungen zu tun hatte. Zum Teil fand die KPA in den Betrieben statt und die Teilnehmenden wurden in die betrieblichen Arbeitsabläufe integriert.

Ein zweiter Erfolgsfaktor besteht in der Bearbeitung von neuen Aufgaben. Dadurch, dass neue und für das kooperierende Unternehmen praxisrelevante Fragestellungen bearbeitet wurden, stießen die KPA-Teilnehmenden mit ihrer Arbeit auf ein anhaltendes Interesse bei den Unternehmen. Ihre Arbeit wurde ernst genommen, da der Betrieb sich mit dem entstehenden Produkt auseinandersetzte. Auf der Seite der Teilnehmenden weckte das Neue den Entdeckergeist und stiftete so gemäß der Zielstellung des entdeckenden und forschenden Lernens Sinn und stärkte den Ehrgeiz und das Engagement.

Drittens, muss der Bezug zum wissenschaftlichen Arbeiten durch die Einbettung in die Welt der Hochschulen genannt werden. Was könnte einem arbeitslosen Akademiker Besseres widerfahren, als sich noch einmal in einer akademischen Umgebung zu bewegen? Akademiker wollen strukturiert verändern und mit ihrer Arbeit Nützliches tun und dafür Anerkennung erhalten. Die Einbindung von Hochschulen bzw. hochschulnahen Einrichtungen in die KPA übte in diesem Zusammenhang eine besondere 
Attraktivität auf die Teilnehmenden aus. Darüber hinaus wurde über das Mitwirken von Trainern und Weiterbildnern der Hochschulen gewährleistet, dass die Bewältigung der betrieblichen Projektaufgaben mithilfe neuster und aktuell gültiger wissenschaftlicher Methoden erfolgen konnte.

Schließlich bildet das selbstständige Arbeiten mit professioneller Unterstützung den vierten Erfolgsfaktor. Die Projektteams haben ihre Aufgabenstellung eigenständig bearbeitet. Sie haben die Arbeit selbst organisiert und einen ganzheitlichen Arbeitsprozess durchlaufen. So konnten die Teilnehmenden praktische Kompetenzen wie Kommunikation, Teamarbeit oder Planung und Organisation erlernen und praktisch ausprobieren. In den verschiedenen Projektphasen wurden die Teams durch Trainer unterstützt. Dabei wurde jedoch nicht in die erarbeiteten Inhalte eingegriffen, sodass sich die KPA-Teilnehmer voll und ganz mit ihrem Produkt identifizieren konnten und sich ein gewisser Produktstolz herausbildete, der über

(6) Weiterführende Informationen sind in der Broschüre „Kreative Projektarbeit - Ein Weg der Qualifizierung für ältere arbeitslose Akademikerinnen, Akademiker und Fachkräfte" festgehalten (http://www.lasa-brandenburg.de/ Ergebnisse.846.0.html). die KPA-Teilnahme hinaus Bestand hatte. Somit wurde das bei den Teilnehmenden aufgebaute Selbstbewusstsein über die Projektphase hinaus erhalten.

Ältere arbeitslose Akademiker stellen mit ihren fachlichen Kenntnissen und Erfahrungen ein wichtiges Fachkräftepotenzial dar, das immer noch häufig außer Acht gelassen wird. Die KPA ist ein möglicher Ansatz, um dieses zu ändern. Sie bietet außerdem eine Struktur, in der die Hochschulen - über ihren Auftrag zur akademischen Lehre und Forschung hinaus - zukünftig verstärkt auch als Weiterbildungsakteur agieren können. ${ }^{\odot}$

\section{AUTOR}

JÖRG JURKEIT, Diplom-Volkswirt, ist Referent im Ministerium für Arbeit, Soziales, Frauen und Familie des Landes Brandenburg. Arbeitsschwerpunkte: Arbeitsmarktförderung für ältere Arbeitnehmer und Langzeitarbeitslose, demografischer Wandel in der Arbeitswelt, sozialer Arbeitsmarkt.

joerg.jurkeit@masf.brandenburg.de 